\title{
Polycystic Ovary Syndrome: Implication for Drug Metabolism on Assisted Reproductive Techniques-A Literature Review
}

\author{
Enrique Reyes-Muñoz $\cdot$ Thozhukat Sathyapalan · Paola Rossetti \\ Mohsin Shah · Min Long · Massimo Buscema - Gaetano Valenti · \\ Valentina Lucia La Rosa (D) - Stefano Cianci · Salvatore Giovanni Vitale
}

Received: August 6, 2018 / Published online: October 11, 2018

(C) The Author(s) 2018

\begin{abstract}
Polycystic ovary syndrome (PCOS) affects $6-10 \%$ of women and could be considered one of the most common endocrine alterations in women of reproductive age. The syndrome is characterized by several hormonal and metabolic alterations, including insulin resistance and hyperandrogenism, which play a severe
\end{abstract}

Enhanced digital features To view enhanced digital features for this article go to https://doi.org/10.6084/ m9.figshare.7159808.

\section{E. Reyes-Muñoz}

Department of Endocrinology, Instituto Nacional de Perinatología Isidro Espinosa de los Reyes, Mexico City, Mexico

T. Sathyapalan

Department of Diabetes and Endocrinology,

University of Hull, Hull, UK

P. Rossetti · M. Buscema

Unit of Diabetology and Endocrino-Metabolic

Diseases, Hospital for Emergency Cannizzaro,

Catania, Italy

M. Shah

Department of Physiology, Institute of Basic

Medical Sciences, Khyber Medical University,

Peshawar, Pakistan

M. Long

Department of Endocrinology, Translational

Research Key Laboratory for Diabetes, Xinqiao

Hospital, Third Military Medical University,

Chongqing, China detrimental role in the patient's fertility. We aimed to offer an overview about drug metabolism in the PCOS population. Nevertheless, we did not find any study that directly compared drug metabolism between PCOS and healthy women. We therefore decided to summarize briefly how hormonal and insulin sensitizer drugs act differently in healthy and PCOS women, who show altered steroidogenesis by theca cells and metabolic imbalance, focusing especially on assisted reproductive techniques. To date, data about drug metabolism in the PCOS population appears to be extremely

G. Valenti · S. G. Vitale

Department of General Surgery and Medical Surgical Specialties, University of Catania, Catania, Italy

V. L. La Rosa $(\bowtie)$

Unit of Psychodiagnostics and Clinical Psychology, University of Catania, Catania, Italy

e-mail: psicolarosa@gmail.com

S. Cianci

Unità Operativa Ginecologia Oncologica,

Dipartimento Scienze della Salute della Donna e del Bambino, Fondazione Policlinico Universitario A. Gemelli, IRCCS, Rome, Italy 
limited. This important gap could have significant implications for therapeutic approaches and future perspectives: the dosage of drugs commonly used for the treatment of PCOS women should be tailored according to each patient's characteristics; we should implement new clinical trials in order to identify the best pharmacologic strategy for PCOS patients undergoing in vitro fertilization (IVF); it would be advisable to create an international expert panel to investigate the drug metabolism in the PCOS population.

Keywords: Assisted reproductive technology; Fertility; Hyperandrogenism; Hyperinsulinemia; Infertility; Insulin resistance; Insulin sensitizers; In vitro fertilization; Oligoanovulation; Polycystic ovary syndrome

\section{INTRODUCTION}

Polycystic ovary syndrome (PCOS) affects $6-10 \%$ of women [1] and could be considered one of the most common endocrine alterations in women of reproductive age. This syndrome is characterized by clinical or biochemical hyperandrogenism, oligo-anovulatory cycles and polycystic ovaries. According to the Rotterdam criteria, it is necessary to have two of the three aforementioned criteria to diagnose PCOS [2]. On the other hand, 10 years ago the Androgen Excess-PCOS (AE-PCOS) Society Task Force stressed the importance of hyperandrogenism in PCOS presentation [3], and 3 years later confirmed the presence of hyperandrogenism together with ovarian dysfunction (oligo-anovulation and/or polycystic features) as diagnostic criteria, notwithstanding the necessity of accurate differential diagnosis for other disorders [1].

The exact pathogenetic mechanisms behind different PCOS features are still unclear; however, numerous studies have deepened our understanding, and the causes are linked to steroidogenesis enzyme function and metabolic disturbance related to insulin resistance. Considering these alterations in PCOS women, several drugs and other metabolically active compounds may have different effects in this population group compared with healthy women without PCOS as a result of hormonal and metabolic imbalance. In this literature review we aim to offer an overview about drug metabolism in women with PCOS.

After extensive literature review we did not find any study that evaluated drug metabolism in PCOS compared with healthy women. For this reason, we decided to summarize briefly different pathogenic mechanisms behind PCOS and how hormonal and insulin sensitizer medications act differently in healthy and PCOS women, especially with respect to assisted reproductive techniques.

\section{METHODS}

We searched the following electronic bibliographic databases: MEDLINE, EMBASE, Global Health, The Cochrane Library (Cochrane Database of Systematic Reviews, Cochrane Central Register of Controlled Trials, Cochrane Methodology Register), Health Technology Assessment Database, and Web of Science (science and social science citation index). We included only manuscripts published in the English language, without time restriction. Titles and/or abstracts of studies retrieved using the search strategy and those from additional sources were screened independently by two authors (V.L.L.R., S.G.V.) to identify studies that potentially meet the inclusion criteria outlined above and fall within the study aims. The full texts of these potentially eligible studies were retrieved and independently assessed for eligibility by other two authors (G.V., S.C.). Any disagreement between them over the eligibility of particular studies was resolved through discussion with a third author (M.B.). A standardised, pre-piloted form was used to extract data from the included studies for assessment of study quality and evidence synthesis. The searches were rerun just before the final analyses, in order to include further studies for inclusion.

On the basis of the aim of the current paper, we first searched "polycystic ovary syndrome AND drug metabolism", "polycystic ovary syndrome AND pharmacokinetics", "polycystic ovary syndrome AND pharmacodynamics": 
from this first search, we did not identify any relevant study comparing directly drug metabolism between PCOS and healthy women. Subsequently, we searched "polycystic ovary syndrome AND substrate" and we found 135 items that were not pertinent to our topic; in addition, searching "pharmacokinetics OR pharmacokinetics AND polycystic ovary syndrome" afforded 173 items, but only one study pertinent to our topic, a study of pregnant women affected by PCOS [4]. Surprisingly, we did not find any study that directly compared drug metabolism between PCOS and healthy women. We therefore decided to summarize briefly how hormonal and insulin sensitizer drugs act differently in healthy and PCOS women, who show intrinsic altered steroidogenesis by theca cells and metabolic imbalance, focusing especially on assisted reproductive techniques.

This article is based on previously conducted studies and does not contain any studies with human participants or animals performed by any of the authors.

\section{Steroidogenesis Alteration in PCOS}

Usually androgens have only been considered as detrimental hormones in oocyte maturation; however, recently their in follicle maturation has been re-evaluated. New evidence showed that androgen, via genomic and non-genomic signal transduction, induced oocyte maturation, especially in the early stage of follicle maturation $[5,6]$. Androgen acts synergistically with folliclestimulating hormone (FSH), modifying steroidogenic enzymes and metabolism in granulosa cells [6]. To confirm these results, treatment of follicles with anti-androgenic drugs was shown to alter and arrest oocyte meiotic maturation. Nevertheless, excess androgen has detrimental effects on oocyte maturation.

Hyperandrogenism occurs in up to $70-80 \%$ women affected by PCOS and it is extremely frequent during adolescence. The aetiology of hyperandrogenism during PCOS is not completely understood. In normal women, androgens are synthesized by ovarian theca cells and adrenal glands, contributing half and half to circulating testosterone during reproductive age
[7]. Interestingly, hyperandrogenism in PCOS appears to be related to dysregulation of steroidogenesis in both ovary and adrenal glands. Luteinising hormone $(\mathrm{LH})$ also plays a pivotal role in the regulation of ovarian androgen production, whereas adrenocorticotropic hormone (ACTH) regulates androgen production by adrenal glands. In addition, several enzymes of the cytochrome P450 family are involved in hormonal balance [8] including cholesterol side-chain cleavage enzyme (P450 scc), aromatase (P450 arom), 3 $\beta$-hydroxysteroid dehydrogenase type II (3ßHSD-II) and steroid acute regulatory protein.

Several studies confirmed the reduction of aromatase activity in ovaries from hyperandrogenic PCOS women with respect to normoandrogenic ones $[9,10]$, leading to a significant reduction of oestradiol/testosterone ratio. Interestingly, aromatase activity is enhanced by oestrogens and inhibited by androgens [10]. One study found a reduction of $3 \beta \mathrm{HSD}-\mathrm{II}$ activity in hyperandrogenic PCOS women compared to normoandrogenic PCOS ones [11]. Other data showed increased 11 $\beta$-hydroxysteroid dehydrogenase II (11 $\beta$-HSDII) activity to convert dehydroepiandrosterone (DHEA) into androstenedione in hyperandrogenic PCOS women compared to normoandrogenic ones and controls [9].

Steroidogenesis is overactive at multiple steps in PCOS, especially concerning 17,20lyase activity [12]: indeed, accumulating evidence suggests that androstenedione/17-OH progesterone ratio in response to gonadotropinreleasing hormone $(\mathrm{GnRH})$ stimulation is lower in PCOS women compared with healthy controls, probably as a result of the lower activity of $17 \alpha$-hydroxylase [13].

Recent data confirmed increased activities of steroidogenic enzymes in hyperandrogenic PCOS women, although the regulation of delta $(\Delta)-4$ and $\Delta-5$ enzymes seems different. On the one hand, the 17 $\alpha$-hydroxylase [14] and 17,20lyase $[8,14]$ activities in the $\Delta-4$ pathway of hyperandrogenic PCOS women lead to higher production rates of $17 \alpha$-hydroxyprogesterone from progesterone, and androstenedione from $17 \alpha$-hydroxyprogesterone, with respect to normoandrogenic PCOS women; on the other hand, 
both hyperandrogenic and normoandrogenic PCOS women have similar activity of the $\Delta-5$ pathway $[9,14]$. In partial agreement with these results, others showed that oestrogens may activate 17,20-lyase, increasing only the production rate of androstenedione ( $\Delta-4$ pathway) [15], or both androstenedione and DHEA $(\Delta-4$ and $\Delta-5$ pathways) [10], even though not all studies confirmed this hypothesis [14]. Nevertheless, after diagnostic criteria standardization, robust data proved that the phosphorylation of serine/ threonine residues, as well as cytochrome $b_{5}$ and cytochrome $c$ allosteric actions were all involved in 17,20 -lyase regulation $[16,17]$. Not surprisingly, the dysregulation of the cytochrome P450 $17 \alpha$-hydroxylase (P450c17 $\alpha$ ) and consequent increase in 17,20-lyase activities were observed only in PCOS.

\section{Hyperinsulinemia in PCOS}

Hyperinsulinemia plays key role in the pathogenesis of PCOS and related hyperandrogenism. In particular, some studies showed a decrease of insulin sensitivity from $35 \%$ to $40 \%[18,19]$. Despite the typical insulin resistance of PCOS, previous studies found that ovarian theca cells had hypersensitivity to insulin stimulation and increase androgen production in response to it [20-22]. This point is particularly debated: some studies showed that insulin alone is able to stimulate testosterone production by cultured theca cells from normal and PCOS patients [22]; conversely, others found that insulin alone is not able to stimulate testosterone production [20]. Similarly, porcine theca cell culture showed increased expression of cytochrome P450 17alpha-hydroxylase/17,20-lyase (CYP17) after insulin and LH stimulation [23], whereas others failed to confirm these results [24].

Interestingly, many studies showed that prolonged suppression of insulin in PCOS women by weight loss [25], diazoxide [26], statins or metformin [27-29] results in significant reduction of androgen production. Diazoxidemediated reduction of insulin level was also found even in lean normoinsulinemic PCOS women [30], but the same did not occur for testosterone levels [31].
The elevated LH in PCOS women is able to stimulate steroidogenesis within theca cells. In addition, theca cells were shown to be abnormally sensitive to LH stimulation, although downregulation of LH receptors may occur [32].

\section{Therapeutic Approaches}

\section{Assisted Reproductive Techniques}

Female infertility has increased in the last decades: recent data from the Centers for Disease Control (CDC) National Survey of family growth show that $6 \%$ of women aged between 15 and 44 years of childbearing age are infertile and $12 \%$ have impaired fertility [33]. Aging is one of the principal factors that play a detrimental role in female fertility as a result of reduced quality of oocytes, ovulatory dysfunction and other gynaecological disorders [34, 35]. Several cohort studies on large populations found that oocyte quality and embryo development may be severely affected by PCOS [36]. In particular, accumulating evidence suggests that the number of small preantral follicles is increased in anovulatory PCOS women: the excess of these types of follicles may be due to accelerated follicle growth and/or prolonged survival (reduced atresia) of small follicles [37]. Interestingly, others found that granulosa cells from ovulatory PCOS women have similar response to FSH with respect to healthy women; conversely, granulosa cells isolated from some small to medium-sized antral follicles obtained from anovulatory PCOS women showed increased oestradiol production in response to FSH and premature responsiveness to $\mathrm{LH}$ $[38,39]$. This process seems to allow overexpression of LH receptors and premature luteinisation of granulosa cells [40], which could be considering a leading cause of the arrest of follicular development in anovulatory PCOS women. Nevertheless, some authors found that follicles from PCOS women undergoing GnRH analogue/FSH stimulation for in vitro fertilization (IVF) show normal luteinisation [40].

Recently, PCOS phenotypes were further investigated and this approach has improved our knowledge about hormonal/metabolic dysregulation, especially in ovulatory/ 
hyperandrogenic PCOS women and ovulatory/ normoandrogenic ones [38]. According to recent evidence, hyperandrogenic women with PCO on ultrasound are at high risk of developing hyperstimulation syndrome during ovulation induction [41]. In addition, ovulatory women with PCO did not show worse IVF/intracytoplasmic sperm injection (ICSI) outcomes (including live birth rate) compared to healthy controls [42].

Some studies found that PCOS women undergoing IVF had lower $\mathrm{FSH}$, the oocyte retrieval was higher and the fertilization rate slightly lower compared with controls, without any significant difference regarding embryo arrest (EA) rate [43], regardless of stimulation protocol used and testosterone level. Although others confirmed that there is no difference in EA, clinical abortion rate in PCOS women seems to be higher than in healthy controls [44].

To date, it is widely accepted that hyperandrogenism, high LH level and insulin resistance may all play a detrimental role in oocyte quality [45-47]: these alterations seem to be particularly pronounced in the zona pellucida of oocytes from PCOS women and this may account, at least in part, for the lower fertilization rate observed during ICSI [48].

However, robust data about this topic are still lacking and the evidence published so far does not allow one to draw a firm conclusion about the best management of hormonal/ metabolic dysregulation in PCOS patients undergoing IVF. Although a recent meta-analysis [49] demonstrates similar pregnancy and live birth rates per cycle (despite an increased cancellation rate, but more oocytes retrieved per retrieval and a lower fertilization rate) in PCOS women undergoing IVF, we truly think that future pharmacologic strategies for assisted reproductive technologies should be tailored according to the patient's characteristics and, most importantly, PCOS phenotype.

\section{Insulin Sensitizer Drugs}

Recent data suggested that the reduction of insulin level with diazoxide or insulin sensitizer drugs such as metformin, thiazolidinediones (PPAR- $\gamma$ agonist) and inositols also reduces hyperandrogenism [50-52]. Because insulin resistance and secondary hyperinsulinemia play an important role in hyperandrogenism, anovulation or irregular cycles, and metabolic alterations in both lean and obese PCOS patients, insulin sensitizers are currently receiving increasing attention for pharmacologic management of this syndrome [53]. Metformin's effect appears to be mediated by reduction of insulin level, both in normal weight and obese women, improving also hyperandrogenism [54-57]. Some authors showed that metformin acts directly on ovarian steroidogenesis in a dose-dependent manner: gonadotropin and insulin stimulate progesterone and oestradiol production by theca cells, whereas metformin inhibits androgen production with no effect on progesterone [57].

Beside metformin, to date inositols have also received increasing attention owing to their safety profile and key role in modulating secondary messenger activity in insulin signal transduction pathways [58]. Inositol is a polyalcohol, it is classified as an insulin sensitizer and it is a secondary messenger in insulin signalling $[58,59]$. We know that two different stereoisomers are currently used in the treatment of PCOS: myo-inositol (MI) and D-chiroinositol (DCI). Both stereoisomers have an insulin-like action in vivo. In humans, accumulating evidence suggests that MI and DCI have clear and beneficial effects on oocyte maturation and metabolic pathways $[59,60]$. Confirming the latter role, others found that DCI levels were about 50\% lower in tissue and urinary samples from patients with type 2 diabetes compared to those in healthy controls [61]. Both MI and DCI were therefore evaluated in PCOS women and shown to ameliorate hormonal and metabolic parameters [62, 63]. Although the last Cochrane systematic review about the topic does not offer a robust recommendation about the best treatment for PCOS among insulin sensitizer medications [64], a recent study did not find any significant difference between metformin and MI in lowering body mass index, ameliorating insulin sensitivity and improving the menstrual cycle [65].

Robust data underline the importance of MI in oocyte differentiation and the possibility to improve IVF outcomes in PCOS women [66-68]: 
in particular, the direct correlation between oocyte quality and follicular fluid concentration of MI was demonstrated both in animal models and humans $[69,70]$. Unfer et al. compared MI with DCI in patients with PCOS, finding that MI had the capacity to ameliorate the quality of oocytes, to decrease the number of immature oocytes and to improve the number of good quality embryos compared to DCI treatment during an ovarian stimulation protocol [67]; others found that in patients under 35 years old, MI plus DCI improved the fertilization rate [68].

Administration of either MI (4 g/day) or DCI ( $1 \mathrm{~g} /$ day) together with folic acid reduced androgens, LH level, LH/FSH ratio and insulin sensitivity in PCOS women [71-73]. MI seems to have the most marked effect on metabolic parameters, whereas DCI seems to have the most marked effect on hyperandrogenism [74-79]. According to several studies, MI is able to increase oocyte quality, reducing the rate of immature oocytes, during IVF [62, 63, 74]. Last but not least, MI seems able to reduce the days of FSH stimulation, oestradiol level before human chorionic gonadotropin administration and risk of hyperstimulation syndrome [63]. Once again, we take the opportunity to underline that future pharmacologic strategies should target insulin resistance selectively according to each patient's characteristics. To date we are able to measure insulin resistance, for example using the homeostasis model assessment, so that we would solicit future tailored treatment of PCOS women.

To date we have a number of studies on insulin sensitizer therapies to assess fertility outcomes in women with PCOS; there were no significant differences in live birth or fetal outcomes to recommend their use in routine clinical practice.

\section{New Possible Targets}

A recent mathematical review of microarray data in patients with PCOS showed that 504 genes are differently expressed, accounting for the altered hormonal/metabolic pathways in this syndrome [80]. Some authors found that the level of serological thymidine kinase 1 (STK1), a protein kinase involved in liver metabolism of metformin, was strictly related to the ovulation response after treatment with metformin and, additionally, the response differs according to PCOS phenotypes [81]. Similarly, other gene expressions may play pivotal roles in addressing altered drug action(s) during PCOS [82]. For example, the adipokine chemerin physiologically binds it receptor, chemochine receptor like-1, and inhibits FSHinduced steroidogenesis; according to recent evidence from a mouse model, chemerin is able to suppress FSH-induced progesterone and oestrogen secretion in preantral follicles and granulosa cells, also inhibiting aromatase expression [83]. Another possible strategy could be to target phosphatidylinositide 3-kinase (PI3K): indeed, recent data showed that the flavonoid quercetin may have a beneficial effect in PCOS by virtue of inhibition of PI3K, which was attributed to a decrease in the expression of CYP17A1 gene, and thereby has a key role in steroidogenesis [84].

\section{CONCLUSIONS}

To date, PCOS could be considered as a multifactorial disease due to concurrent hormonal and metabolic alterations that influence each other. Insulin resistance represents one of the key elements of the syndrome and causes multiple effects: on the one hand it increases androgen production by theca cells, on the other hand it alters the follicle maturation and reduces oocyte quality. On the basis of this scenario, insulin resistance rapidly became an important target and several trials found beneficial effects of insulin sensitizer drugs on hormonal, metabolic and even reproductive outcomes in the PCOS population. Despite these important therapeutic improvements, we should consider that the PCOS population may have significantly different responses to several classes of drugs as a result of a unique hormonal/metabolic environment. The situation could be even more complicated if we consider that PCOS could not be identified as a single syndrome, but as different phenotypes 
according to clinical and biochemical characteristics. To date, data about drug metabolism in the PCOS population appears to be almost nonexistent or at least extremely limited. This important gap in the literature could have significant implications for therapeutic approaches and future perspectives: first of all, the dosage of drugs commonly used for the treatment of PCOS women should be tailored according to each patient's characteristics; second, we should implement new clinical trials in order to identify the best pharmacologic strategy for PCOS patients undergoing IVF; finally, it would be advisable to create an international expert panel to investigate the drug metabolism in the PCOS population.

In addition, we take the opportunity to solicit new research aimed to also explore how different classes of drugs influence the epigenetic up- and downregulation of genes related to steroidogenesis and insulin transduction signalling, especially through genome-wide association studies (GWAS).

\section{ACKNOWLEDGEMENTS}

Funding. No funding or sponsorship was received for this study or publication of this article.

Authorship. All named authors meet the International Committee of Medical Journal Editors (ICMJE) criteria for authorship for this article, take responsibility for the integrity of the work as a whole, and have given their approval for this version to be published.

Disclosures. Enrique Reyes-Muñoz, Thozhukat Sathyapalan, Paola Rossetti, Mohsin Shah, Min Long, Massimo Buscema, Gaetano Valenti, Valentina Lucia La Rosa, Stefano Cianci and Salvatore Giovanni Vitale have nothing to disclose. The authors alone are responsible for the content and writing of the paper.

Compliance with Ethics Guidelines. This article is based on previously conducted studies and does not contain any studies with human participants or animals performed by any of the authors.

Data Availability. Data sharing is not applicable to this article as no datasets were generated or analyzed during the current study.

Open Access. This article is distributed under the terms of the Creative Commons Attribution-NonCommercial 4.0 International License (http://creativecommons.org/licenses/ by-nc/4.0/), which permits any noncommercial use, distribution, and reproduction in any medium, provided you give appropriate credit to the original author(s) and the source, provide a link to the Creative Commons license, and indicate if changes were made.

\section{REFERENCES}

1. Azziz R. Polycystic ovary syndrome. Obstet Gynecol. 2018;132:321-36.

2. Rotterdam ESHRE/ASRM-Sponsored PCOS consensus workshop group. Revised 2003 consensus on diagnostic criteria and long-term health risks related to polycystic ovary syndrome (PCOS). Hum Reprod. 2004;19:41-7.

3. Azziz R, Carmina E, Dewailly D, et al. Positions statement: criteria for defining polycystic ovary syndrome as a predominantly hyperandrogenic syndrome: an Androgen Excess Society guideline. J Clin Endocrinol Metab. 2006;91:4237-45.

4. de Oliveira Baraldi C, Lanchote VL, de Jesus Antunes N, et al. Metformin pharmacokinetics in nondiabetic pregnant women with polycystic ovary syndrome. Eur J Clin Pharmacol. 2011;67:1027-33.

5. Lutz LB, Jamnongjit M, Yang WH, Jahani D, Gill A, Hammes SR. Selective modulation of genomic and non genomic androgen responses by androgen receptor ligands. Mol Endocrinol. 2003;17:1106-16.

6. Lenie S, Smitz J. Functional AR signalling is evident in an in vitro mouse follicle culture bioassay that encompasses the most stages of folliculogenesis. Biol Reprod. 2009;80:685-95.

7. Piltonen $\mathrm{T}$, Koivunen $\mathrm{R}$, Morin-Papunen $\mathrm{L}$, et al. Ovarian and adrenal steroid production: regulatory role of/LHHCG. Hum Reprod. 2002;17:620-4. 
8. Qin K, Rosenfield RL. Role of cytochrome P450c17 in polycystic ovary syndrome. Mol Cell Endocrinol. 1998;145:111-21.

9. De Medeiros SF, Barbosa JS, Yamamototo MM. Comparison of steroidogenic pathway among normoandrogenic and hyperandrogenic polycystic ovary syndrome patients and normal cycling woman. J Obstet Gynaecol Res. 2014;41:254-63.

10. Chen J, Shen S, Tan Y, et al. The correlation of aromatase activity and obesity in women with and without polycystic ovary syndrome. J Ovar Res. 2015;8:11.

11. Doi SA, Al-Zaid M, Towers PA, et al. Steroidogenetic alteration and adrenal androgen excess in PCOS. Steroid. 2006;71:751-9.

12. Rosenfield R, Ehrmann D. The pathogenesis of polycystic ovary syndrome (PCOS): the hypothesis of PCOS as functional ovarian hyperandrogenism revisited. Endocr Rev. 2016;37:467-520.

13. Rosenfield RL, Barnes RB, Ehrmann DA. Studies of the nature of 17-hydroxyprogesterone hyperresonsiveness to gonadotropin-releasing hormone agonist challenge in functional ovarian hyperandrogenism. J Clin Endocrinol Metab. 1994;79:1686-92.

14. Medeiros SF, Gil-Junior AB, Barbosa JS, et al. New insights into steroidogenesis in normo- and hyperandrogenic polycystic ovary syndrome patients. Arq Bras Endocrinol Metabol. 2013;57:437-44.

15. Ditkoff EC, Fruzzetti F, Chang L, et al. The impact of estrogen on adrenal androgen sensitivity and secretion in polycystic ovary syndrome. J Clin Endocrinol Metab. 1995;80:603-7.

16. Auchus RJ, Lee TC, Miller WL. Cytochrome b5 augments the 17,20-lyase activity of human P450c17 without direct electron transfer. J Biol Chem. 1998;273:3158-65.

17. Lin D, Black SM, Nagahama Y, et al. Steroid 17 alpha-hydroxylase and 17,20-lyase activities of P450c17: contribution of serine106 and P450 reductase. Endocrinology. 1993;132:2498-506.

18. Book CB, Dunaif A. Selective insulin resistance in the polycystic ovary syndrome. J Clin Endocrinol Metab. 1999;84:3110-6.

19. Dunaif A, Wu X, Lee A, et al. Defects in insulin receptor signalling in vivo the polycystic ovary syndrome (PCOS). Am J Physiol Endocrinol Metab. 2001;281:E392-9.

20. Munir I, Yen H, Geller D, et al. Insulin augmentation of 17alpha-hydroxylase activity is mediated by phosphatidyl inositol 3-kinase but not extracellular signal-regulated kinase-1/2 in human ovarian theca cells. Endocrinology. 2004;145:175-83.

21. Wu XK, Zhou SY, Liu JX, et al. Selective ovary resistance to insulin signaling in women with polycystic ovary syndrome. Fertil Steril. 2003;80:954-65.

22. Nestler J, Jakubowicz D, De Vargas A, et al. Insulin stimulates testosterone biosynthesis by human theca cells from women with polycystic ovarian syndrome by activating its own receptor and using inositol glycan mediators as the signal transduction system. J Clin Endocrinol Metab. 1998;83:2001-5.

23. Zhang G, Garmey JC, Veldhuis JD. Interactive stimulation by luteinizing hormone and insulin of the steroidogenic acute regulatory (StAR) protein and 17alpha-hydroxylase/17,20-lyase (CYP17) genes in porcine theca cells. Endocrinology. 2000;141:2735-42.

24. Cadagan D, Khan R, Amer S. Thecal cell sensitivity to luteinizing hormone and insulin in polycystic ovarian syndrome. Reprod Biol. 2016;16:53-60.

25. Holte J, Bergh $\mathrm{T}$, Berne $\mathrm{C}$, Wide L, Lithell $\mathrm{H}$. Restored insulin sensitivity but persistently increased early insulin secretion after weight loss in obese women with polycystic ovary syndrome. J Clin Endocrinol Metab. 1995;80:2586-93.

26. Nestler JE, Barlascini CO, Matt DW, et al. Suppression of serum insulin by diazoxide reduces serum testosterone levels in obese women with polycystic ovary syndrome. J Clin Endocrinol Metab. $1989 ; 68: 1027-32$.

27. Nestler JE, Jakubowicz DJ. Decreases in ovarian cytochrome P450c17 alpha activity and serum free testosterone after reduction of insulin secretion in polycystic ovary syndrome. $\mathrm{N}$ Engl $\mathrm{J}$ Med. 1996;335:617-23.

28. Sathyapalan T, Kilpatrick ES, Coady AM, Atkin SL. The effect of atorvastatin in patients with polycystic ovary syndrome: a randomized double-blind placebo-controlled study. J Clin Endocrinol Metab. 2009;94:103-8.

29. Sathyapalan T, Cho LW, Kilpatrick ES, Coady AM, Atkin SL. A comparison between rimonabant and metformin in reducing biochemical hyperandrogenaemia and insulin resistance in patients with polycystic ovary syndrome (PCOS): a randomized open-label parallel study. Clin Endocrinol (Oxf). 2008;69:931-5.

30. Nestler JE, Singh R, Matt DW, et al. Suppression of serum insulin level by diazoxide does not alter serum testosterone or sex hormone-binding 
globulin levels in healthy, nonobese women. Am J Obstet Gynecol. 1990;163:1243-6.

31. Baillargeon JP, Nestler JE. Commentary: polycystic ovary syndrome: a syndrome of ovarian hypersensitivity to insulin? J Clin Endocrinol Metab. 2006;91:22-4.

32. Ehrmann DA, Barnes RB, Rosenfield RL. Polycystic ovary syndrome as a form of functional ovarian hyperandrogenism due to dysregulation of androgen secretion. Endocr Rev. 1995;16:322-53.

33. Chandra A, Copen CE, Stephen EH. Infertility and impaired fecundity in the United States, 1982-2010: data from the National Survey of Family Growth. Natl Health Stat Report. 2013;67:1-18, $1 \mathrm{p}$ following 19.

34. Baird DT, Collins J, Egozcue J, et al. Fertility and ageing. Hum Reprod Update. 2005;11:261-76.

35. Aquino CI, Nori SL. Complementary therapy in polycystic ovary syndrome. Transl Med UniSa. 2014;9:56-65.

36. Qiao J, Feng HL. Extra- and intra-ovarian factors in polycystic ovary syndrome: impact on oocyte maturation and embryo developmental competence. Hum Reprod Update. 2011;17:17-33.

37. Webber LJ, Stubbs S, Stark J, et al. Formation and early development of follicles in the polycystic ovary. Lancet. 2003;362:1017-21.

38. Willis DS, Watson $\mathrm{H}$, Mason HD, et al. Premature response to luteinizing hormone of granulosa cells from anovulatory women with polycystic ovary syndrome: relevance to mechanism of anovulation. J Clin Endocrinol Metab. 1998;83:3984-91.

39. Mason HD, Willis DS, Beard RW, et al. Estradiol production by granulosa cells of normal and polycystic ovaries: relationship to menstrual cycle history and concentrations of gonadotropins and sex steroids in follicular fluid. J Clin Endocrinol Metab. 1994;79:1355-60.

40. Foong SC, Abbott DH, Zschunke MA, et al. Follicle luteinization in hyperandrogenic follicles of polycystic ovary syndrome patients undergoing gonadotropin therapy for in vitro fertilization. J Clin Endocrinol Metab. 2006;91:2327-33.

41. Fauser BC, Diedrich K, Devroey P, et al. Predictors of ovarian response: progress towards individualized treatment in ovulation induction and ovarian stimulation. Hum Reprod Update. 2008;14:1-14.

42. Costello MF, Chew CY, Lindsay K, et al. Effect of polycystic ovaries on in vitro fertilization and intracytoplasmatic sperm injection treatment outcome. Asian Pacific J Reprod. 2016;5:182-7.

43. Yin B, Hao H, Wei D, et al. Patients with polycystic ovary syndrome have successful embryo arrest. Int J Clin Exp Med. 2015;8:6247-51.

44. Kodama H, Fukuda J, Karube $\mathrm{H}$, et al. High incidence of embryo transfer cancellations in patients with polycystic ovarian syndrome. Hum Reprod. 1995;10:1962-7.

45. Marci R, Senn A, Dessole S, et al. A low-dose stimulation protocol using highly purified folliclestimulating hormone can lead to high pregnancy rates in in vitro fertilization patients with polycystic ovaries who are at risk of a high ovarian response to gonadotropins. Fertil Steril. 2001;75:1131-5.

46. Tarlatzis BC, Grimbizis G. The significance of high follicular-phase luteinizing hormone levels in the treatment of women with polycystic ovarian syndrome by in vitro fertilization. J Assist Reprod Genet. 1997;14:1-4.

47. Stadtmauer LA, Toma SK, Riehl RM, et al. Metformin treatment of patients with polycystic ovary syndrome undergoing in vitro fertilization improves outcomes and is associated with modulation of the insulin-like growth factors. Fertil Steril. 2001;75:505-9.

48. Hwang JL, Seow KM, Lin YH, et al. IVF versus ICSI in sibling oocytes from patients with polycystic ovarian syndrome: a randomized controlled trial. Hum Reprod. 2005;20:1261-5.

49. Heijnen EM, Eijkemans MJ, Hughes EG, et al. A meta-analysis of outcomes of conventional IVF in women with polycystic ovary syndrome. Hum Reprod Update. 2006;12:13-21.

50. Baillargeon JP. Use of insulin sensitizers in polycystic ovarian syndrome. Curr Opin Investig Drugs. 2005;6:1012-22.

51. Dokras A, Saini S, Gibson-Helm M, et al. Gaps in knowledge among physicians regarding diagnostic criteria and management of polycystic ovary syndrome. Fertil Steril. 2017;107(1380-1386):e1.

52. Bloomgarden ZT, Futterweit W, Poretsky L. Use of insulin-sensitizing agents in patients with polycystic ovary syndrome. Endocr Pract. 2001;7:279-86.

53. Legro RS, Arslanian SA, Ehrmann DA, et al. Diagnosis and treatment of polycystic ovary syndrome: an Endocrine Society clinical practice guideline. J Clin Endocrinol Metab. 2013;98:4565-92.

54. Kurzthaler D, Hadziomerovic-Pekic D, Wildt L, Seeber BE. Metformin induces a prompt decrease in 
LH-stimulated testosterone response in women with PCOS independent of its insulin-sensitizing effects. Reprod Biol Endocrinol. 2014;12:98.

55. Rice S, Pellatt L, Ramanathan K, et al. Metformin inhibits aromatase via an extracellular signal-regulated kinase-mediated pathway. Endocrinology. 2009;150:4794-801.

56. Tan S, Hahn S, Benson S, et al. Metformin improves polycystic ovary syndrome symptoms irrespective of pre-treatment insulin resistance. Eur J Endocrinol. 2007; 157:669-76.

57. Yilmaz M, Biri A, Karakoç A, et al. The effects of rosiglitazone and metformin on insulin resistance and serum androgen levels in obese and lean patients with polycystic ovary syndrome. J Endocrinol Invest. 2005;28:1003-8.

58. Papaleo E, Unfer V, Baillargeon JP, et al. Contribution of myo-inositol to reproduction. Eur J Obstet Gynecol Reprod Biol. 2009;147:120-3.

59. Bizzarri M, Fuso A, Dinicola S, Cucina A, Bevilacqua A. Pharmacodynamics and pharmacokinetics of inositol(s) in health and disease. Expert Opin Drug Metab Toxicol. 2016;12:1181-96.

60. Laganà AS, Rossetti $\mathrm{P}$, Buscema $\mathrm{M}$, et al. Metabolism and ovarian function in PCOS women: a therapeutic approach with inositols. Int J Endocrinol. 2016;2016:6306410.

61. Asplin I, Galasko G, Larner J. Chiro-inositol deficiency and insulin resistance: a comparison of the chiro-inositol- and the myo-inositol-containing insulin mediators isolated from urine, hemodialysate, and muscle of control and type II diabetic subjects. Proc Natl Acad Sci USA. 1993;90:5924-8.

62. Paul C, Laganà AS, Maniglio $\mathrm{P}$, et al. Inositol's and other nutraceuticals' synergistic actions counteract insulin resistance in polycystic ovarian syndrome and metabolic syndrome: state-of-the-art and future perspectives. Gynecol Endocrinol. 2016;32:431-8.

63. Laganà AS, Sapia F, La Rosa VL, Vitale SG. Comment on "Inositols: from physiology to rational therapy in gynecological clinical practice". Expert Opin Drug Metab Toxicol. 2016;12:1527.

64. Tang T, Lord JM, Norman RJ, Yasmin E, Balen AH. Insulin-sensitising drugs (metformin, rosiglitazone, pioglitazone, D-chiro-inositol) for women with polycystic ovary syndrome, oligo amenorrhoea and subfertility. Cochrane Database Syst Rev. 2012;5:CD003053.

65. Fruzzetti F, Perini D, Russo M, Bucci F, Gadducci A. Comparison of two insulin sensitizers, metformin and myo-inositol, in women with polycystic ovary syndrome (PCOS). Gynecol Endocrinol. 2017;33:39-42.

66. Carlomagno G, Unfer V, Roseff S. The D-chiroinositol paradox in the ovary. Fertil Steril. 2011;95:2515-6.

67. Unfer V, Carlomagno G, Rizzo P, Raffone E, Roseff $\mathrm{S}$. Myo-inositol rather than D-chiro-inositol is able to improve oocyte quality in intracytoplasmic sperm injection cycles. A prospective, controlled, randomized trial. Eur Rev Med Pharmacol Sci. 2011;15:452-7.

68. Colazingari S, Treglia M, Najjar R, Bevilacqua A. The combined therapy myo-inositol plus D-chiro-inositol, rather than D-chiro-inositol, is able to improve IVF outcomes: results from a randomized controlled trial. Arch Gynecol Obstet. 2013;288:1405-11.

69. Chiu TT, Rogers MS, Law EL, et al. Follicular fluid and serum concentrations of myo-inositol in patients undergoing IVF: relationship with oocyte quality. Hum Reprod. 2002;17:1591-6.

70. Chiu TT, Rogers MS, Briton-Jones C, et al. Effects of myo-inositol on the in vitro maturation and subsequent development of mouse oocytes. Hum Reprod. 2003;18:408-16.

71. Laganà AS, Barbaro L, Pizzo A. Evaluation of ovarian function and metabolic factors in women affected by polycystic ovary syndrome after treatment with D-chiro-inositol. Arch Gynecol Obstet. 2015;291:1181-6.

72. Pizzo A, Laganà AS, Barbaro L. Comparison between effects of myo-inositol and D-chiro-inositol on ovarian function and metabolic factors in women with PCOS. Gynecol Endocrinol. 2014;30:205-8.

73. Genazzani AD, Lanzoni C, Ricchieri F, et al. Myoinositol administration positively affects hyperinsulinemia and hormonal parameters in overweight patients with polycystic ovary syndrome. Gynecol Endocrinol. 2008;24:139-44.

74. Vitale SG, Rossetti P, Corrado F, et al. How to achieve high-quality oocytes? The key role of myoinositol and melatonin. Int $\mathrm{J}$ Endocrinol. 2016;2016:4987436.

75. Muscogiuri G, Palomba S, Laganà AS, et al. Inositols in the treatment of insulin-mediated diseases. Int J Endocrinol. 2016;2016:3058393.

76. Laganà AS, Pizzo A. Authors' reply to: "Empiric" inositol supplementation in normal-weight noninsulin resistant women with polycystic ovarian disease: from the absence of benefit to the potential 
adverse effects. Arch Gynecol Obstet. 2015;291:959-60.

77. Gulino FA, Leonardi E, Marilli I, et al. Effect of treatment with myo-inositol on semen parameters of patients undergoing an IVF cycle: in vivo study. Gynecol Endocrinol. 2016;32:65-8.

78. Muscogiuri G, Palomba S, Laganà AS, et al. Current insights about inositol isoforms, mediterranean and ketogenic diets for polycystic ovary syndrome: from bench to beside. Curr Pharm Des. 2016;22:5554-7.

79. Laganà AS, Rossetti $\mathrm{P}$, Sapia F, et al. Evidence-based and patient-oriented inositol treatment in polycystic ovary syndrome: changing the perspective of the disease. Int $\mathrm{J}$ Endocrinol Metab. 2017; 15:e43695.

80. Mohamed-Hussein ZA, Harun S. Construction of a polycystic ovarian syndrome (PCOS) pathway based on the interactions of PCOS-related proteins retrieved from bibliomic data. Theor Biol Med Model. 2009;6:18.

81. Legro RS, Barnhart HX, Schiaff WD. Ovulatory response to treatment of polycystic ovary syndrome is associated with a polymorphism in the STK11 gene. J Clin Endocrinol Metab. 2008;93:792-800.

82. Goldenberg N, Glueck CJ. Is pharmacogenomics our future? Metformin, ovulation and polymorphism of the STK11 gene in polycystic ovary syndrome. Pharmacogenomics. 2008;9:1163-5.

83. Wang Q, Kim JY, Xue K, et al. Chemerin, a novel regulator of follicular steroidogenesis and its potential involvement in polycystic ovarian syndrome. Endocrinology. 2012;153:5600-11.

84. Shah KN, Patel SS. Phosphatidylinositide 3-kinase inhibition: a new potential target for the treatment of polycystic ovarian syndrome. Pharm Biol. 2016;54:975-83. 